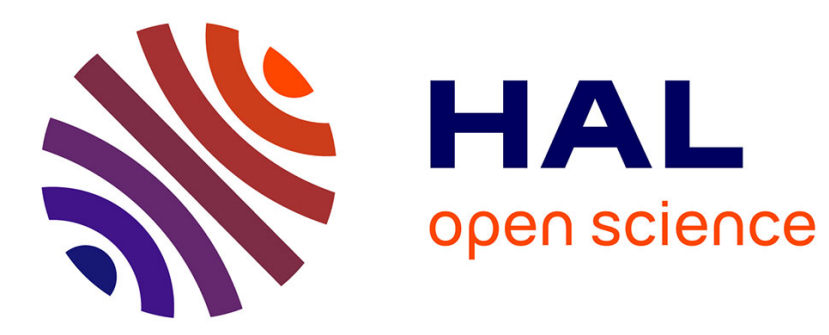

\title{
Imagerie ultrasonore haute résolution
}

\author{
M. Berson, G. Feuillard, S. Diridollou, M. Letiecq, L. Pourcelot
}

\section{To cite this version:}

M. Berson, G. Feuillard, S. Diridollou, M. Letiecq, L. Pourcelot. Imagerie ultrasonore haute résolution. Journal de Physique IV Proceedings, 1994, 04 (C5), pp.C5-1285-C5-1288. 10.1051/jp4:19945285 . jpa-00252997

\section{HAL Id: jpa-00252997 https://hal.science/jpa-00252997}

Submitted on 1 Jan 1994

HAL is a multi-disciplinary open access archive for the deposit and dissemination of scientific research documents, whether they are published or not. The documents may come from teaching and research institutions in France or abroad, or from public or private research centers.
L'archive ouverte pluridisciplinaire HAL, est destinée au dépôt et à la diffusion de documents scientifiques de niveau recherche, publiés ou non, émanant des établissements d'enseignement et de recherche français ou étrangers, des laboratoires publics ou privés. 


\title{
Imagerie ultrasonore haute résolution
}

\author{
M. BERSON*, G. FEUILLARD, S. DIRIDOLLOU, M. LETIECQ et L. POURCELOT
}

GIP Ultrasons, Laboratoire de Biophysique Médicale, Faculté de Médecine, 2 Bis Boulevard Tonnellé, 37032 Tours cedex, France

* Unité INSERM 316, Laboratoire de Biophysique Médicale, Faculté de Médecine, 2 Bis Boulevard Tonnellé, 37032 Tours cedex, France

\begin{abstract}
High resolution acoustical imaging involves hard constraints for the ultrasonic probes which are used: a high resonance center frequency, a very wide bandwidth, a good sensitivity and a strong focusing of the beam. The system which is described uses two kinds of focused transducer: a very broad band $17 \mathrm{MHz}$ center frequency transducer (PZT), and a $45 \mathrm{MHz}$ copolymer transducer. The focal distances are respectively $7 \mathrm{~mm}$ and $6 \mathrm{~mm}$ in water. The movement of the transducer provides a $6 \mathrm{~mm}$ wide and $5 \mathrm{~mm}$ deep echographic cross-section with at the rate of 15 images/s. The resolution is respectively at $17 \mathrm{MHz}$ and $45 \mathrm{MHz}, 80 \mu \mathrm{m}$ and $60 \mu \mathrm{m}$ in axial and $250 \mu \mathrm{m}$ and $60 \mu \mathrm{m}$ in lateral in the focal zone. The device was tested on fantoms in water and in vivo with applications in Dermatology.
\end{abstract}

\section{INTRODUCTION}

L'imagerie ultrasonore à haute résolution (au maximum $0,1 \mathrm{~mm}$ en axial et 0,2 à $0,3 \mathrm{~mm}$ en latéral) répond à des besoins réels dans plusieurs domaines de l'exploration du corps humain par ultrasons: Dermatologie, Ophtalmologie, Exploration Intra-Vasculaire et IntraCavitaire, Microchirurgie. Elle peut également être utilisée dans l'industrie en contrôle nondestructif (joints de colle, couches de peinture, micro-soudures...).

Une bonne résolution latérale est assez aisément obtenue avec une fréquence élevée associée à une focalisation du faisceau.

En ce qui concerne la résolution axiale, le problème est plus complexe. En effet, une très grande résolution axiale implique l'utilisation d'échos très brefs pour la formation de l'image échographique. Il en résulte deux conditions pour le transducteur: une fréquence centrale de résonance élevée (au moins $15 \mathrm{MHz}$ ) et surtout une bande passante très large (réponse impulsionnelle brève). Ce problème conduit généralement à un compromis délicat entre bande passante et sensibilité et à des difficultés technologiques qui augmentent avec la fréquence. Un autre problème réside dans le choix du matériau piézoélectrique constituant le transducteur. Pour les fréquences dites basses (15 à $30 \mathrm{MHz})$, les céramiques ferroélectriques (PZT: plomb zirconate titanate) sont généralement employées [1]. Les fréquences hautes (supérieures à $30 \mathrm{MHz}$ ) sont souvent le domaine des films plastiques polymères (PVDF) ou copolymères [P(VDF-TrFE)] malgré leur assez faible sensibilité [2]. En effet, le coefficient de couplage électromécanique est très faible pour les polymères $\left(\mathrm{k}_{t}=0,1\right)$. Il est beaucoup plus 
acceptable $\left(k_{t}=0,3\right)$ pour les copolymères. De plus, ces matériaux sont intéressants pour leur faible impédance acoustique ( $\mathrm{Z}=4$ à $5 \mathrm{MRayl}$ ) et leur grande facilité de mise en oeuvre.

En ce qui concerne plus spécialement l'exploration de la peau, les premiers travaux ont été menés avec des fréquences de l'ordre de $10 \mathrm{MHz}$ et des appareils non spécifiques qui ne permettaient pas des résultats suffisants [3]. Récemment, des dispositifs spécialement adaptés et beaucoup plus performants sont apparus [4] [5].

Dans cet article nous décrivons un système d'imagerie haute résolution temps réel (15 images/s) et présentons différents résultats obtenus in vitro et in vivo.

\section{APPAREILLAGE}

\section{1) Sonde}

Dans un système d'imagerie, la sonde est une partie importante et dans cette sonde, le capteur ultrasonore est l'élément essentiel. Nous utilisons deux types de capteur. Le premier type concerne un capteur basse fréquence (fréquence centrale $17 \mathrm{MHz}$ ) (figure 1a) qui a été réalisé en collaboration avec la société Vermon (Tours). Ses dimensions sont les suivantes: diamètre $6 \mathrm{~mm}$ et longueur sans connecteur $12 \mathrm{~mm}$. Ce capteur est composé d'un absorbant arrière (backing), d'un transducteur (disque plat de $3 \mathrm{~mm}$ de diamètre) en céramique piézoélectrique (PZT) et d'une lentille acoustique qui joue également le rôle de lame adaptatrice. Grâce à l'absorbant optimisé, le transducteur est très fortement amorti (bande passante de $22 \mathrm{MHz}$ à $-6 \mathrm{~dB}$ ), tout en gardant une sensibilité suffisante.

La focalisation du faisceau, assurée par la lentille acoustique procure une zone focale centrée à environ $7 \mathrm{~mm}$ de la surface du capteur ultrasonore pour une propagation dans l'eau ou les tissus du corps humain. La largeur théorique du faisceau à $-6 \mathrm{~dB}$ dans le plan focal est de $0,180 \mathrm{~mm}$.

Le second capteur (figure 1b) développé au laboratoire a une fréquence centrale de $45 \mathrm{MHz}$ et il est réalisé avec un disque de plastique copolymère [P(VDF-TrFE)] (Atochem Penwalt) de $3,7 \mathrm{~mm}$ de diamètre et de $17 \mu \mathrm{m}$ d'épaisseur. Ce disque est collé à l'aide d'une colle conductrice (Epotek $410 \mathrm{LV}$ ) sur l'extrémité usinée en forme de calotte sphérique (rayon de courbure $6 \mathrm{~mm}$ ) d'un cylindre en cuivre (impédance acoustique $36 \mathrm{MRayl}$ ) de $8 \mathrm{~mm}$ de longueur et de $3,7 \mathrm{~mm}$ de diamètre. Ce capteur a une bande passante à $-6 \mathrm{~dB}$ de $28 \mathrm{MHz}$ et une largeur théorique $\mathrm{du}$ faisceau à $-6 \mathrm{~dB}$ dans le plan focal de $0,063 \mathrm{~mm}$.

Le capteur ultrasonore est placé dans la sonde (figure 2) à l'extrémité d'un support pour effectuer un balayage sectoriel. La longueur du support à partir de l'axe de rotation est suffisamment grande pour que la présentation de l'image sous une forme rectangulaire ne provoque qu'une déformation négligeable. La sonde de forme cylindrique $(3 \mathrm{~cm}$ de diamètre, $16 \mathrm{~cm}$ de longueur) réalise ainsi des coupes échographiques de $6 \mathrm{~mm}$ de largeur et de $5 \mathrm{~mm}$ de profondeur à une cadence de 15 par seconde.

\section{2) Electronique}

Cette partie comprend de façon classique un ensemble d'émission et de réception et a une bande passante supérieure à celle du capteur afin de ne pas détériorer la résolution axiale.

Le circuit d'émission est réalisé avec un transistor MOSFET de puissance et la chaine de réception comporte un préamplificateur à gain variable et à bande passante large (Plessey SL610, $85 \mathrm{MHz}$ ), suivi d'un amplificateur logarithmique avec une dynamique de $60 \mathrm{~dB}$. Un tel amplificateur non linéaire permet d'effectuer la compression nécessaire pour une présentation correcte de toute la gamme des échos reçus. La présentation des images est faite sous forme analogique (dispositif de visualisation de type XYZ) ou sur l'écran d'un microordinateur après numérisation. 


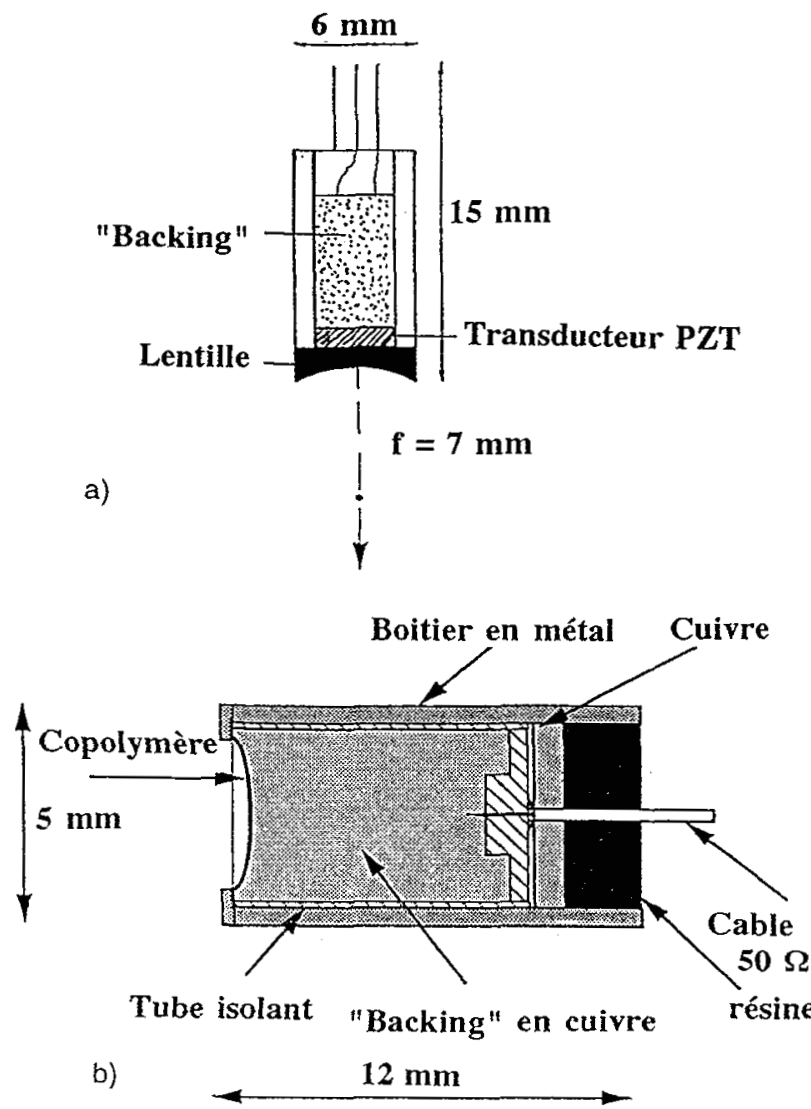

Figure 1: a) capteur $17 \mathrm{MHz}$

b) capteur $45 \mathrm{MHz}$

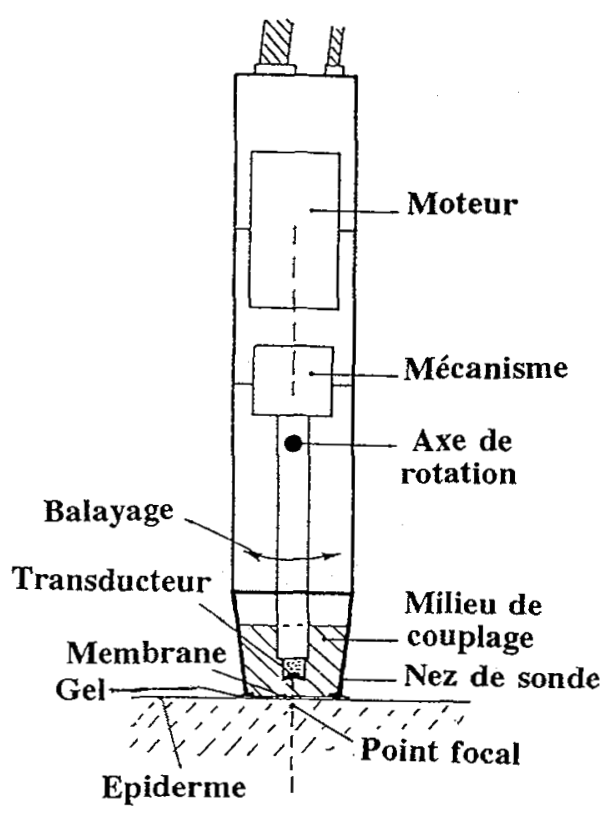

Figure 2: Schéma de principe de la sonde.

\section{RÉSULTATS}

La figure 3a présente l'écho fourni par le capteur de fréquence centrale $17 \mathrm{MHz}$ après réflexion sur un réflecteur plan parfait, placé dans le plan focal. La "traine" de cet écho est très peu importante et autorise une résolution axiale de l'ordre de $80 \mu \mathrm{m}$ dans l'eau. L'écho obtenu de façon identique avec le capteur de fréquence central $45 \mathrm{MHz}$ apparait sur la figure $3 \mathrm{~b}$. Cet écho qui correspond à une bande passante relative à $-6 \mathrm{~dB}$ de $51 \%$ permet une résolution axiale de $60 \mu \mathrm{m}$.

Les explorations de la peau (normale et pathologique) sont menées en routine dans le Service de Dermatologie de l'Hôpital Trousseau à Tours. Un gel spécifique est alors nécessaire entre la membrane du "nez de sonde" (figure 2) et la peau pour assurer le contact acoustique. Des images de peau normale obtenues avec le capteur $45 \mathrm{MHz}$ sont présentées sur la figure 4 . On peut y distinguer l'image de la membrane de la sonde (1), l'interface gelépiderme (2), le derme (3), l'interface derme-hypoderme (4), des ensembles glande sébacéepoil (5) et des petits vaisseaux (6) 


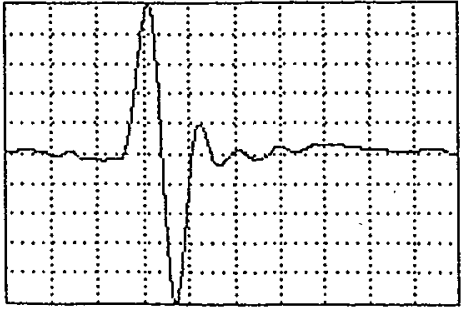

40. $n s / d i c$

a)

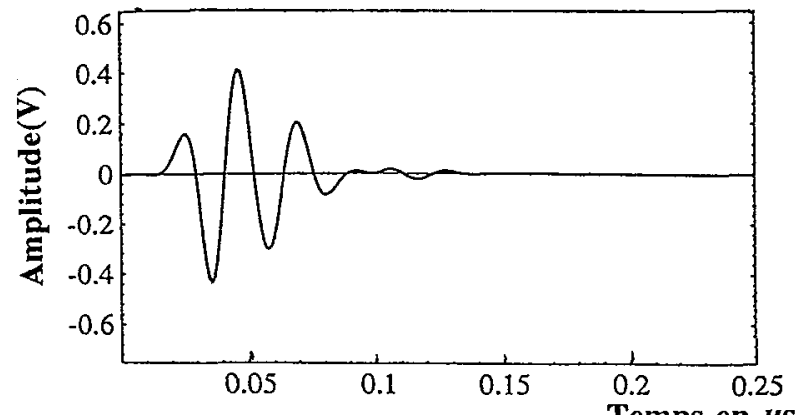

b)

Figure 3: a) écho obtenu avec le capteur $17 \mathrm{MHz}$; b)écho obtenu avec le capteur $45 \mathrm{MHz}$.

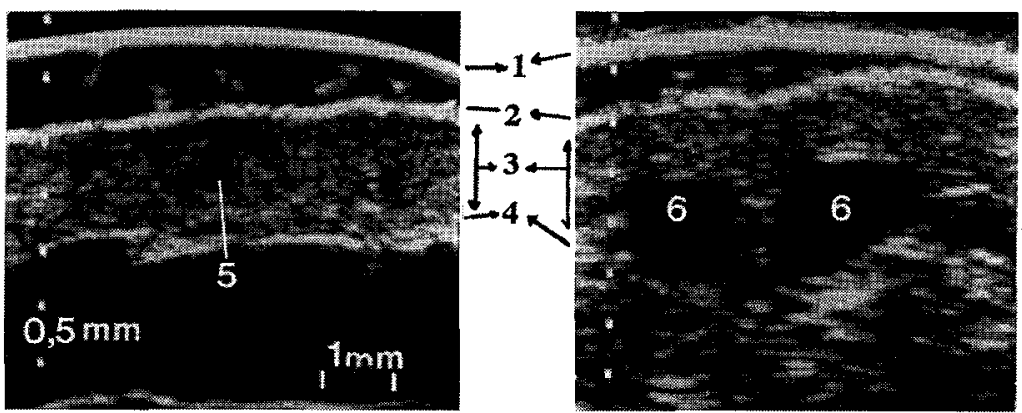

Figure 4: Image de peau normale: (1) membrane ; (2) interface gel-épiderme ; (3) derme ; (4) interface derme-hypoderme ; (5) glande sébacée-poil ; (6) vaisseau.

\section{CONCLUSION}

Cet article montre les possibilités de l'imagerie acoustique dans la gamme de fréquence 15-50 MHz. L'optimisation des performances implique d'une part, le maintien d'une très large bande passante quelque soit la fréquence centrale (optimisation de la résolution axiale) et d'autre part, la réalisation de capteurs plus complexes permettant d'obtenir une longueur de zone focale plus élevée (plusieurs $\mathrm{mm}$ ).

\section{RÉFÉRENCES}

[1] FORSTER F.S., RYAN L.K., and TURNBULL D.H. Characterisation of lead zirconate titanate ceramics for the use in miniature high frequency $(20-80 \mathrm{MHz})$ transducer. IEEE Transactions on UFFC 38-5 (1991) 446-453.

[2] HURMILA S., STUBB H., PITKANEN J., LAHDENPERA K., PENTTINEN A., SUORSA V., and TAURIAINEN A. Ultrasonic transducer using PVDF. Ferroelectrics 115 (1991) 267-278.

[3] QUERLEUX B., LEVEQUE J.L., and DE RIGAL J. In vivo cross sectional ultrasonic imaging of human skin. Dermatologica 177 (1988) 332-337.

[4] BERSON M., VAILLANT L., PATAT F., and POURCELOT L. High resolution real time ultrasonic scanner. Ultrasound Med. Biol. 18 (1992) 471-478. 